$\mathrm{n}=1$ commented that the forms give insight into patient presentations and management

Conclusion. Doctors routinely prescribe Z-drugs and benzodiazepines, and would generally consider Haloperidol as a second line over Promethazine (while nurses had a slight preference for requesting Promethazine over Haloperidol). The role of 12 lead electro-cardiogram monitoring would require further exploration in separate audits, as both Promethazine and Haloperidol can cause QTc interval prolongation [4,5].

Doctors most commonly cited expectations by nursing staff as the main driver for PRN medication prescription. Profound differences were present with regards to rationale behind PRN medication use when comparisons between doctors and nurses selfreports were made. The majority of nurses cited ward atmosphere and patient dependence/expectation as main drivers, whereas a minority of doctors shared those views. This represents a concerning disconnect between professionals, although it can be explained by the higher proportion of time ward nurses spend on mental health wards and in direct patient care. Nursing staff, being the dispensers of medication, would also likely be the main professionals contacted for the request of PRN medication by patients.

Nuanced views were given to the role of psychological redirection. This was shared between doctors and nurses, although many cited concerns about nursing staff shortages leading to a possible overreliance on PRN medication. A minority of doctors ( $\mathrm{n}=$ 2) would recommend psychological redirection after first line rapid tranquilisation was exhausted. The counterargument being that someone admitted onto a ward tacitly implies a high level of acuity and reduced appropriateness of psychological techniques.

Hypnotics most commonly being requested likely reflects the difficult nature to initiate and maintain sleep is an acute ward setting.

On review of the Round 2 results indicate that doctors and nurses agree that the new system is safer although more time consuming. Concerns were raised about rapid tranquilisation and immediate emergencies, although the revised policy would allow for the verbal order policy to be followed with a digital order in these circumstances. This was clarified via further communication with relevant parties.

The changes were more received more positively by doctors than nurses, with some nurses opting for the older system if possible. It was also raised that this may be putting up barriers for out of hours prescriptions, although the required information is arguably succinct and only requests vital information for safe prescribing. Further exploration of these concerns would be indicated. The Round 2 results were limited by the low sample size compared to the first round.Despite the limitations and concerns about the new system, digitising the system allows for further audits and studies to utilize much more robust methods of measuring out of hours prescriptions than self-reported measures employed in the initial rounds. Although they may not be directly compared to findings of this report, future baselines can be established and compared to in an objective manner.

Future Rounds

Proposed: To design and clearly display information on commonly requested medication by patients, empowering them to make more informed decisions on the medications they request. This could be in the form of leaflets patients could take or posters on areas where patients receive medication. One example is that Zopiclone is a very commonly requested medication on an as required basis although patients may not be as aware of the risks associated with chronic use.

Proposed: To design and clearly display information on psychologically informed techniques in patient areas such distress tolerance and sleep hygiene. This would be on mental health sites which do not currently display this information. To measure impact on PRN medication dispensation.

Proposed: Further exploration of patient perceived ward environment and measures that can be implemented to reduce anxiety/ insomnia associated with inpatient admission.

Proposed: Exploration of proportion of inpatient initiated PRN medication progresses to long term use in the community (largely focused on hypnotics and benzodiazepines).

\section{Dr QI - A quality improvement (QI) approach to designing and delivering QI training}

Deepa Bagepalli Krishnan ${ }^{1 \star}$, Victor Ohize ${ }^{2}$ and Luke Baumber ${ }^{2}$

${ }^{1}$ Institute of Mental Health, University of Nottingham,

Nottinghamshire Healthcare NHS Foundation Trust and

${ }^{2}$ Nottinghamshire Healthcare NHS Foundation Trust

${ }^{\star}$ Corresponding author.

doi: 10.1192/bjo.2021.475

Aims. To develop and implement a QI training programme for trainees, Trust grade doctors and Consultants in Nottinghamshire Healthcare NHS Trust (NHFT) to enable them to deliver change in practice through acquisition of new knowledge and practical application of skills in QI projects using Model for Improvement.

Background. QI is crucial to improve patient care. Doctors are uniquely placed to input into patient safety and service delivery of healthcare. The skills required to be future clinical leaders and undertake improvement work are not innate and formal teaching and support is required.

What is DrQI?

DrQI is a trainee-led QI teaching programme developed in collaboration with Trainees improving patient safety through QI (TIPSQI) in North West deanery.

Method. A pre-implementation survey amongst doctors in NHFT in February 2019 (33 responses) suggested that 90\% of doctors were interested in learning QI and about $48 \%$ preferred face-face workshops with support from the QI team.

A list of change ideas were created using a driver diagram with QI education and project support identified as key primary drivers. PDSA cycles

Nine interactive workshops teaching key QI concepts (based on model for improvement) in NHFT, training more than 100 doctors. A workshop in Derbyshire Healthcare NHS Foundation Trust (70 doctors) and Nottingham University Hospital (20 doctors). Workshops were continually adapted based on qualitative and quantitative feedback. Different formats were tried including virtual sessions, game-based and problem-based learning approaches using small group activities.

Result. Pre-course and post-course questionnaires were used to assess change in understanding of individual components of QI methodology (SMART Aim, Driver diagram, PDSA cycles, outcome and process measures and run charts). Mean pre-course self-assessment score collated from seven QI workshops in NHFT (2019-2020) was 3.3 and mean post-course score was 7.68, showing an improvement in understanding of QI methodology.

Participants were asked to score the relevance (8.4) and quality of teaching (8.4) and the support from the QI team (7.4) on a scale of $1-10(1=$ poor and $10=$ excellent $)$. Additional free text feedback was obtained to help us improve the teaching programme.

Conclusion. Collaborative leadership trainee-led initiative to increase the QI capacity. A bottom up approach to complement the top down approach from the Trust QI team. Future steps include further collaboration and expansion of the scheme to other Trusts, Train the trainer sessions and building a network of QI champions. 
Prescribing and monitoring high dose and combined antipsychotics in community mental health team

Dipanjan Banerjee ${ }^{1 \star}$, Tracey Green ${ }^{2}$ and Umama Khan ${ }^{3}$

${ }^{1}$ East Sussex Healthcare NHS Trust; ${ }^{2}$ St Marys Hospital and ${ }^{3}$ St Marys Hospital

${ }^{\star}$ Corresponding author.

doi: 10.1192/bjo.2021.476

Aims. The aim was to establish the prevalence of prescription of combined and high dose antipsychotics in the community mental health team and to see if such patients were being offered regular monitoring as advised by NICE guidelines.

Background. The use of high dose antipsychotic treatment (HDAT) should be in line with the recommendations of the Royal College of Psychiatrists Consensus statement on high dose antipsychotic Treatment. Such treatment should be initiated only when standard treatments have failed. As high dose prescribing of antipsychotic medications can potentially harm than benefit causing serious side effects and sudden death. Close monitoring and documentation are required. Also, reviewing these patients at regular intervals is recommended.

Method. A retrospective audit of 50 case notes of patients currently on antipsychotics was done. Case notes were selected randomly from a pool of 300 plus patients under the care of Isle of Wight NHS Trust in the HoNos Cluster 11 and 12 care pathways. Data were gathered on patients' demographics, diagnosis, medication monitoring. Data were analysed and discussed with consultant psychiatrist and senior mental health pharmacist. Patients who were not on any antipsychotic medications were not included in the audit.

Result. $90 \%$ of the patients were on single antipsychotic (45 out of 50 ), $4.45 \%$ (2 out of 45 )were above BNF recommended dose. 10\% (5) patients were prescribed combined antipsychotics. $40 \%$ (2)of them were above BNF recommended maximum dose. A total of $8 \%$ ( 4 out of 50 ) patients were on above BNF recommended maximum dose. All the patients on high dose antipsychotics had a clear plan documented in the system. Documented monitoring of Full blood count was found in $75 \%$, blood glucose in $50 \%$, lipid in $75 \%$, cardiac monitoring (Electrocardiogram or ECG) in $0 \%$, physical health monitoring in $0 \%$

Conclusion. As far as we know, this is the first time an audit has been done on the patients under the Community Mental Health Team on high dose antipsychotic treatment. The data showed areas of good practice as the majority of the patients were on monotherapy, and all the patients on a high dose or combined antipsychotics had clear management plans outlined in their notes. However, the audit also highlighted areas that currently need improvements such as regular monitoring of the patients on high dose and combined antipsychotics. It should also be clearly documented, recorded, and reviewed at six-monthly intervals.

\section{Co-morbid gambling disorder in a local drug and alcohol service: an audit to determine prevalence}

John Barker ${ }^{1 \star}$, Ruta Rele ${ }^{1}$, Charlotte Cartwright ${ }^{2}$ and Bethany Dinsdale-Young ${ }^{3}$

${ }^{1}$ Sheffield Health and Social Care NHS Foundation Trust; ${ }^{2}$ The University of Sheffield and ${ }^{3}$ The University of Sheffield ${ }^{\star}$ Corresponding author.

\section{doi: 10.1192/bjo.2021.477}

Aims. National surveys show that over 56\% of adults in England gamble annually, and of those surveyed, $0.5 \%$ were problem gamblers, equating to 300,000 problem gamblers at any point. The prevalence of problem gambling in patients with a substance misuse disorder ranges from $20.5 \%$ to $55 \%$.

The audit aims to improve the care of patients with comorbid substance misuse and gambling disorder by assessing the extent to which the service currently enquires about and records problem gambling in its patient cohort.

It is hypothesised that as no formal recording process is in place locally, this information will not be recorded systematically and in a way that is easily retrievable by the service.

The audit will allow the service to assess whether changes need to be made to the initial assessment pathways into treatment for substance-related disorders to adequately record this information so that further assessment and onward referral can take place.

Method. All active patients $(n=2824)$ within the service had both their electronic initial assessments and their entire electronic notes screened for terms such as 'betting' and 'gambling' and this was recorded using an Excel spreadsheet. Prevalence rates across the teams (opiates, non-opiates and alcohol) were then calculated.

Result. The results showed that $0 \%$ of patients had any entries in their initial screening noting any gambling activity. Further scrutiny of the records revealed that only 3.5\% (n-99) had ever discussed gambling with a worker in any of the services.

Conclusion. The majority $(n=52)$ of patients who had discussed gambling only had one positive search result, suggesting this was not followed-up in a systematic fashion. Recommendations are to revise the common assessment pro-forma to include a validated brief screening tool (lie/bet), where one positive answer triggers a further assessment with an appropriate clinician for consideration of referral to the local NHS gambling service.

Improving patient waiting times and quality of care by arranging access to notes from a neighbouring trust

Georgios Basdanis* and Cormac Fenton

Mental Health Liaison Team, St Thomas' Hospital, South London and Maudsley NHS Foundation Trust

${ }^{\star}$ Corresponding author.

doi: $10.1192 /$ bjo.2021.478

Aims. We aim to improve waiting times in the Emergency Department and improve the overall quality of care of out-of-area patients by arranging for the liaison team to have access to the electronic notes system of a neighbouring trust.

Method. St Thomas' Hospital is located in south London, right opposite the City of Westminster. As a result, approximately $20 \%$ of patients we see in mental health liaison are from that locality. Given that they belong to a different trust, we do not have access to their notes, which leads to a delay in trying to establish whether they are known to local mental health services. Often, staff are reluctant to divulge information. When information is shared, it is often late and/or incomplete. We approached the Chief Clinical Information Officer and Head of Information Governance from Central and North West London (CNWL) NHS Foundation Trust. We held weekly meetings which included both IT departments. Our IT had to install the electronic notes application (SystmOne) on our computers and open relevant firewall ports. The information is access through an NHS Smartcard, therefore CNWL had to authorise read-only Smartcard profiles for every member of the liaison team. A quick reference guide was created for all staff that would be using the new application. The system went live on 21 January 2021.

Result. We audited patient outcomes in December 2020 and February 2021 for initial comparison. In December 2020, the 